\title{
Angina de Ludwig e suas complicações: estudo de caso de paciente internado em UTI de um hospital público na região norte do Brasil
}

Angina de Ludwig and its complications: case study of a patient admitted to the ICU of a public hospital in the northern region of Brazil

La angina de Ludwig y sus complicaciones: estudio de caso de un paciente ingresado en la UCI de un hospital público de la región norte de Brasil

Kamyla Souza de Vasconcelos ${ }^{1}$, Messias Froes da Silva Junior ${ }^{2}$, Nely Cristina Medeiros Caires ${ }^{1 *}$.

\section{RESUMO}

Objetivo: Relatar o caso de paciente imunocomprometido, internado em UTI pública na região norte do Brasil e as complicações sistêmicas causada por infecção odontogênica. Detalhamento do caso: Paciente do sexo masculino, 28 anos, deu entrada no Pronto Socorro, com a presença de aumento de volume em região submandibular bilateralmente, com projeção da língua anteriormente, dor e limitação da abertura bucal. Ao histórico médico, paciente portador de Leucemia Linfoblástica Aguda (LLA tipo B comum). Em região mandibular direita extra-oral havia abscesso subjacente comprometendo a pele. $\mathrm{Na}$ região cervical, observouse necrose tecidual até região torácica. Paciente foi internado em UTI, e após a avaliação do cirurgião-dentista, observou-se a presença de resto radicular do elemento 46. O diagnóstico final foi definido como abscesso, angina de Ludwig com evolução para mediastinite e fasceíte necrosante. Foi realizado exodontia do elemento 46, suporte médico, antibioticoterapia e avaliação frequente do paciente pelo cirurgião-dentista. Considerações finais: $O$ cirurgião-dentista é de suma importância em equipe multidisciplinar e ambiente hospitalar, no que tange à prevenção, condução e tratamento odontológico a fim de se evitar complicações graves e comprometimentos sistêmicos no paciente internado.

Palavras-chave: Angina de Ludwig, Odontólogos, Diagnóstico bucal.

\begin{abstract}
Objective: To report the case of an immunocompromised patient admitted to a public ICU in northern Brazil and the systemic complications caused by odontogenic infection. Case details: A 28-year-old male patient was admitted to the Emergency Room with the presence of swelling in the submandibular region bilaterally, with anterior projection of the tongue, pain and limitation of mouth opening. Medical history, patient with Acute Lymphoblastic Leukemia (common type B ALL). In the extra-oral right mandibular region, there was an underlying abscess compromising the skin. In the cervical region, tissue necrosis up to the thoracic region was observed. The patient was admitted to the ICU, and after evaluation by the dentist, the presence of root remains of element 46 was observed. The final diagnosis was defined as abscess, Ludwig's angina with evolution to mediastinitis and necrotizing fasciitis. Extraction of element 46, medical support, antibiotic therapy and frequent patient assessment by the dentist were performed. Final considerations: The dentist is of paramount importance in a multidisciplinary team and in a hospital environment, with regard to prevention, management and dental treatment in order to avoid serious complications and systemic impairments in hospitalized patients.
\end{abstract}

Keywords: Ludwig's Angina, Dentists, Diagnosis oral.

1 Universidade Paulista (UNIP), Manaus - AM. *E-mail: nelycaires@gmail.com

${ }^{2}$ Colégio Brasileiro de Cirurgiões (CBC), Manaus - AM.

SUBMETIDO EM: 6/2021

ACEITO EM: 6/2021

PUBLICADO EM: 7/2021 


\section{RESUMEN}

Objetivo: Informar el caso de un paciente inmunodeprimido ingresado en una UCI pública del norte de Brasil y las complicaciones sistémicas por infección odontogénica. Detalle del caso: Paciente varón de 28 años que ingresa en Urgencias con presencia de tumefacción en región submandibular bilateralmente, con proyección anterior de lengua, dolor y limitación de la apertura bucal. Historia clínica, paciente con leucemia linfoblástica aguda (LLA tipo B común). En la región mandibular derecha extraoral, había un absceso subyacente que comprometía la piel. En la región cervical se observó necrosis tisular hasta la región torácica. El paciente ingresó en la $\mathrm{UCl}$, y luego de la evaluación odontológica se observó la presencia de restos radiculares del elemento 46. El diagnóstico final se definió como absceso, angina de Ludwig con evolución a mediastinitis y fascitis necrosante. Se realizó extracción del elemento 46, soporte médico, antibioterapia y valoración frecuente del paciente por parte del odontólogo. Consideraciones finales: El odontólogo es de suma importancia en un equipo multidisciplinar y en un entorno hospitalario, en lo que respecta a la prevención, el manejo y el tratamiento odontológico para evitar complicaciones graves y deficiencias sistémicas en los pacientes hospitalizados.

Palabras clave: Angina de Ludwig, Odontólogos, Diagnóstico bucal.

\section{INTRODUÇÃO}

Angina de Ludwig é um processo infeccioso grave, poli microbiano, de evolução rápida e apresenta alto índice de mortalidade. Tal condição pode comprometer os espaços submandibulares, sublinguais e submentoniano. Dentre os sinais e sintomas destacam-se: sintomatologia dolorosa, aumento de volume em região cervical, disfagia, trismo, edema do assoalho bucal, protrusão lingual, febre, linfadenopatia e calafrios. A etiologia é na maioria das vezes odontogênica, porém abscessos amigdalianos também são descritos na literatura como uma possível causa (CAMARGOS FM, et al., 2016; DIB JE, et al., 2016; BAEZ-PRAVIA OV, et al., 2017).

Considerada uma urgência terapêutica, seu diagnóstico e abordagem precoces devem ser conduzidos a fim de melhorar o prognóstico do caso. O tratamento consiste em intervenção cirúrgica agressiva e antibioticoterapia sistêmica. Inúmeras complicações graves podem iniciar- se a partir deste quadro, dentre elas destaca-se a Mediastinite. À medida que o processo evolui, o mesmo pode atingir tanto áreas cervicais, quanto a glote, até chegar ao Mediastino, causando assim, a Mediastinite aguda, que mesmo sendo rara, possui uma taxa de mortalidade que varia de $40 \%$ a 60\% (CAMARGOS FM, et al., 2016; FREIRE FFP, et al., 2014).

A Mediastinite Aguda é um processo inflamatório e infeccioso e poli microbiano do tecido conjuntivo do mediastino. Apresenta baixa incidência, mas elevada mortalidade, devido ao reconhecimento muitas vezes tardio desta condição. Tem como fator etiológico primário, na maioria das vezes, uma infecção odontogênica. A infecção tem origem nos planos das fáscias da cabeça e do pescoço, e alcança o mediastino, por meio das fáscias cervicais, podendo também em $70 \%$ dos casos, ocorrer a propagação da infecção via espaço faríngico lateral do espaço retro visceral (retro faríngico), estendendo-se inferiormente ao mediastino (FREIRE FFP, et al., 2014; SOYLU E, et al., 2019).

Correlacionada à Angina de Ludwig e Mediastinite, a Fasceíte Necrosante (FN) é uma condição considerada rara, sendo caracterizada pela necrose extensa dos tecidos moles e formação gasosa no tecido subcutâneo da região cérvico-facial. Geralmente encontra-se associada a pacientes com imunossupressão sistêmica, embora possa acometer também pacientes saudáveis (SOYLU E, et al., 2019). A mortalidade relatada na literatura varia de $13 \%$ a $76 \%$, sendo influenciada pela precocidade do diagnóstico, abordagem cirúrgica e doenças associadas (FERNANDES BR, et al., 2017).

Para que não haja agravantes e prejuízos a saúde e condição sistêmica do paciente se faz necessário um diagnóstico precoce e um plano de tratamento que inclua: desbridamento cirúrgico de todo o tecido necrótico, internação do paciente em ambiente hospitalar, para acompanhamento e realização de antibioticoterapia de amplo espectro (SPREADBOROUGH P, et al., 2016). 
Todas estas condições listadas acima, representam uma preocupação na área de saúde pública, devido ao alto grau de morbimortalidade e altos custos para a realização do tratamento, mesmo que a incidência destas infecções tenha diminuído ao longo dos anos (CAMARGOS FM, et al., 2016; FREIRE FFP, et al., 2014).

Muitos desses pacientes necessitam de assistência hospitalar, e a presença do Cirurgião Dentista (CD) na equipe multidisciplinar é de suma importância; não somente nos estágios avançados das infecções, mas também na manutenção do equilíbrio da cavidade bucal para a prevenção de infecções, no diagnóstico das alterações bucais, além de ser coadjuvante na terapêutica médica (BLUM DFC, et al., 2018; SILVA EA, et al., 2020).

A avaliação das condições orais em pacientes internados em UTI é de extrema importância. Sabe-se que as comorbidades e complicações que levam esses pacientes à internação se somam às possibilidades de adquirir infecção nosocomial durante períodos prolongados de internação. Além disto, o paciente pode sofrer alterações orais decorrentes de doenças sistêmicas ou do uso de medicamentos e ventilação mecânica invasiva (KIYOSHI-TEO H e BLEGEN M, 2015).

A leucemia é subdividida clínica e patologicamente em grandes grupos, dentre os quais, estão suas formas agudas e crônicas. A forma aguda caracteriza-se por um aumento rápido no número de células imaturas do sangue, que faz com que a medula óssea seja incapaz de reproduzir células sanguíneas saudáveis. Já a forma crônica caracteriza-se pelo aumento excessivo no número de células maduras anormais da série branca do sangue (ANTONINI MF, et al., 2018)

A Leucemia pode ainda ser classificada de acordo com o tipo de célula afetada: os linfoblastos, resultando em uma doença denominada leucemia linfoblástica, ao passo que, quando afeta as células granulocíticas e monocíticas, são denominadas leucemias mieloides (MORAES ES, et al., 2017).

A LLA é a neoplasia maligna mais comum na infância e adolescência, apresentando-se frequentemente quimiossensível, alcançando uma taxa de sobrevida em cerca de $90 \%$ quando implementado o regime terapêutico ideal. Representam um terço de todos os cânceres infantis, sendo que $75 \%$ dos pacientes pediátricos sofrem de Leucemia Linfoblástica Aguda (LLA), que pode ser de origem das células B ou T (BATH AA, et al., 2020).

As causas da Leucemia são desconhecidas e incertas, embora possa ser associada à exposição à radiação, anomalias cromossômicas, lesões químicas e infecções virais, podendo ser causa da combinação de fatores ambientais e genéticos. Os sinais mais comuns em cavidade oral são anormalidades gengivais como hipertrofia e sangramento, hemorragia, palidez na mucosa e ulceração, entre outros (LOBÃO DS, et al., 2008).

A partir do exposto, apresentamos o relato de caso de Angina de Ludwig com complicações sistêmicas severas em paciente portador de LLA. O tratamento foi realizado em ambiente hospitalar com intervenção e acompanhamento do cirurgião-dentista junto à equipe multiprofissional.

\section{DETALHAMENTO DO CASO}

O presente relato foi autorizado pelos responsáveis através de Termo de Consentimento Livre e Esclarecido, além de ter sido submetido e aprovado pelo Comitê de Ética em Pesquisa (CEP), parecer CAAE: 98088818.5.0000.5512.

Paciente A.A.F, 28 anos, sexo masculino, melanoderma, foi internado na UTI de um hospital público na região norte do Brasil. Ao exame clínico intra e extraoral notou-se a presença de aumento de volume em região submandibular bilateralmente, com projeção da língua anteriormente, dor e limitação da abertura bucal e presença de resto radicular o elemento 46, além de higiene oral deficiente. Em região mandibular direita havia presença de fístula extraoral comprometendo a pele. Em região cervical, observou-se necrose tecidual até região torácica. 
Realizado os exames laboratoriais, o resultado do hemograma demonstrou dentre outros dados: 3.23 milhões/mm3 de hemácias (Valor de Referência HOMEM/ADULTO: 4.2 a 5.9 milhões/ $\mu \mathrm{L}$ ), hematócrito 28\% (Valor de Referência HOMEM/ADULTO: 45 a $50 \%$ ), e glicemia em Jejum Normal (Valor de Referência: inferior a $99 \mathrm{mg} / \mathrm{dL}$ ) de 146mg/dl (PNCQ, 2017). Embora os valores estivessem alterados quando comparados aos valores de referência, já que o paciente possuía anemia em função da LLA; o paciente encontrava-se hemodinamicamente estável. Diante do quadro clínico apresentado pelo paciente no momento da internação, havia necessidade de conduta emergencial, rápida e precisa.

O paciente foi assistido pela equipe multidisciplinar do hospital, tendo sido solicitada a avaliação do Cirurgião-Dentista e conduta para o caso. O diagnóstico final da condição clínica do paciente foi definido como sendo Abscesso de origem odontogênica, Angina de Ludwig com evolução para Mediastinite e Fasceíte Necrosante (Figura 1).

Figura 1 - Fasceite Necrosante.

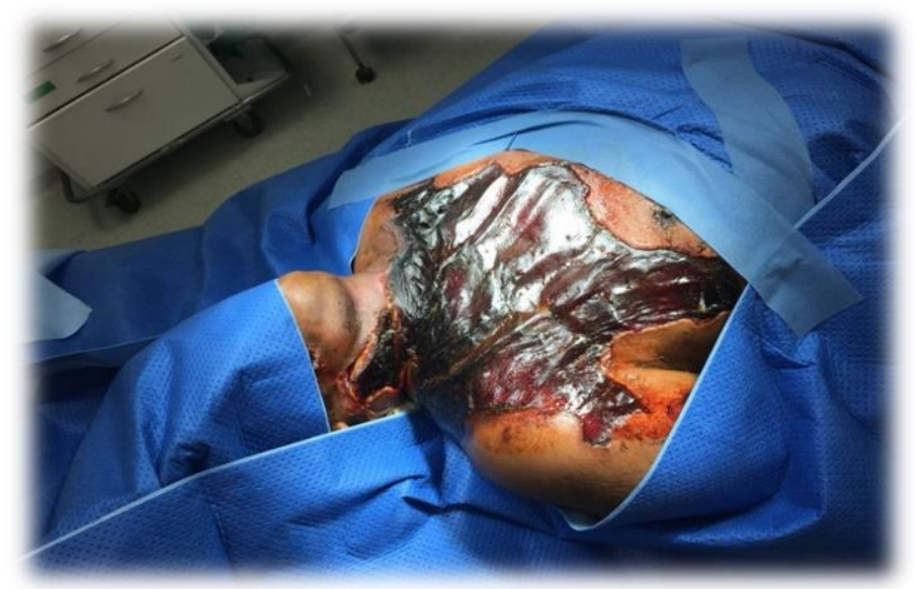

Fonte: Vasconcelos KS, et al., 2021.

A equipe médica iniciou o tratamento com clindamicina (1800 $\mathrm{mg} / \mathrm{dia})$ e também com gentamicina (180mg/dia). E posteriormente amplo desbridamento cirúrgico, com retirada de tecido necrótico (Figura 2).

Figura 2 - Remoção tecido necrótico e desbridamento da ferida.

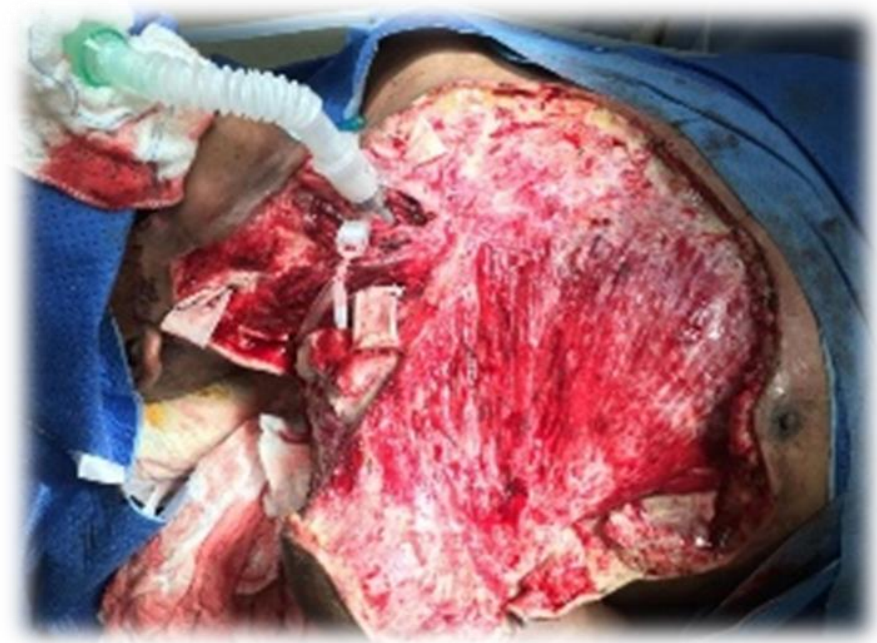

Fonte: Vasconcelos KS, et al., 2021.

A etiologia do abscesso era um resto radicular do elemento 46 e após avaliação, constatou-se a necessidade de um planejamento adequado optando-se pela intervenção cirúrgica, com remoção do elemento em questão em sessão única pela equipe odontológica (Figura 3). 
Figura 3 - Procedimento cirúrgico odontológico de exodontia dente 46, resto radicular.

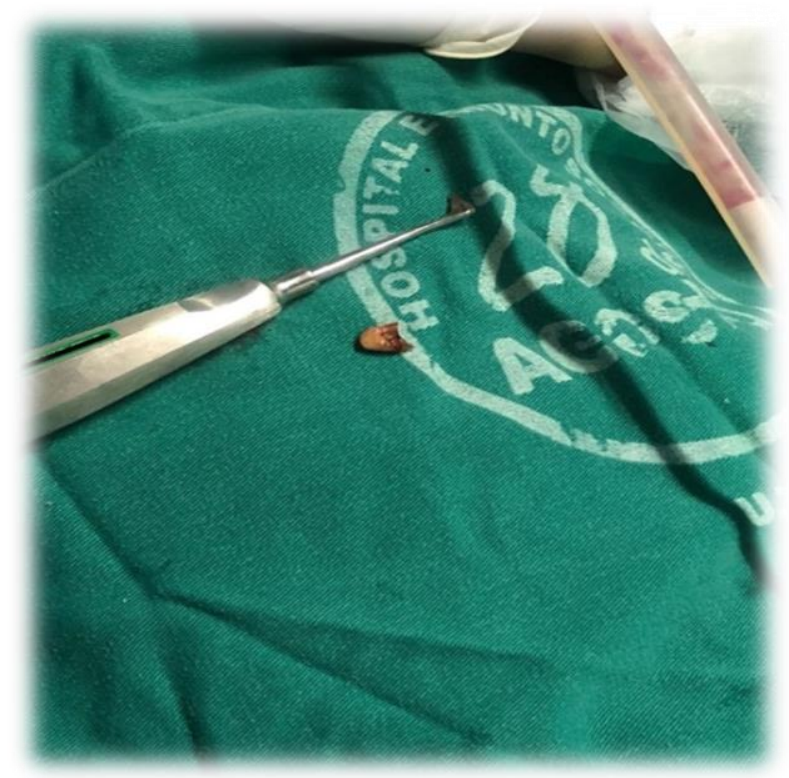

Fonte: Vasconcelos KS, et al., 2021.

No dia da realização do procedimento, o paciente encontrava-se compensado hemodinamicamente, TQT (Traqueostomizado) e sedado com Fentanil 6,6 Mg/Kg /H + Propofol $2 \mathrm{Mg} / \mathrm{Kg} / \mathrm{H}$.

Antes do procedimento, para controle bacteriano, foi realizada uma higienização da cavidade oral com Gluconato de Clorexidina a $0,12 \%$ (Reymer $\AA$ ). Este procedimento foi mantido após a exodontia pela equipe de Enfermagem, sendo realizado de 12/12 horas conforme protocolo proposto pelo Departamento de Odontologia da Associação de Medicina Intensiva Brasileira (AMIB) (2013).

Decorridas 24 horas após a realização do procedimento odontológico, observou-se discreta melhora no quadro clínico do paciente, já com início da cicatrização de fístula extraoral (Figura 4).

Figura 4 - 24 horas após procedimento cirurgico odontológico de exodontia dente 46.

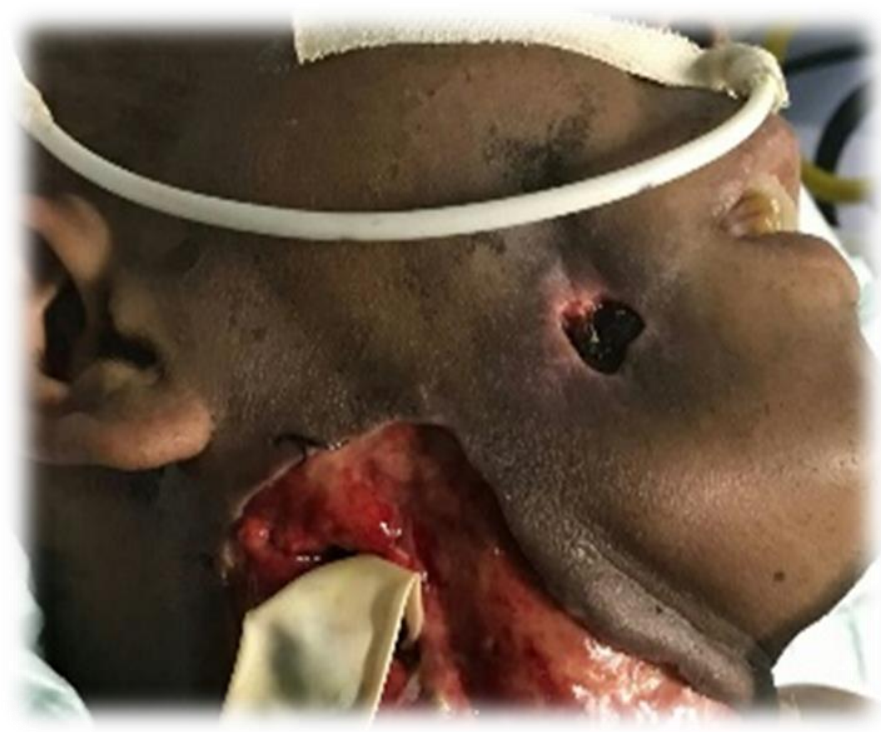

Fonte: Vasconcelos KS, et al., 2021.

O paciente permaneceu hospitalizado por 3 meses, aguardando a realização do enxerto e, neste período, manteve-se com boa evolução clínica e assistência odontológica diária (Figura 5 e Figura 6). 
Figura 5 - 30 dias após a realização do procedimento cirúrgico odontológico de exodontia dente 46.

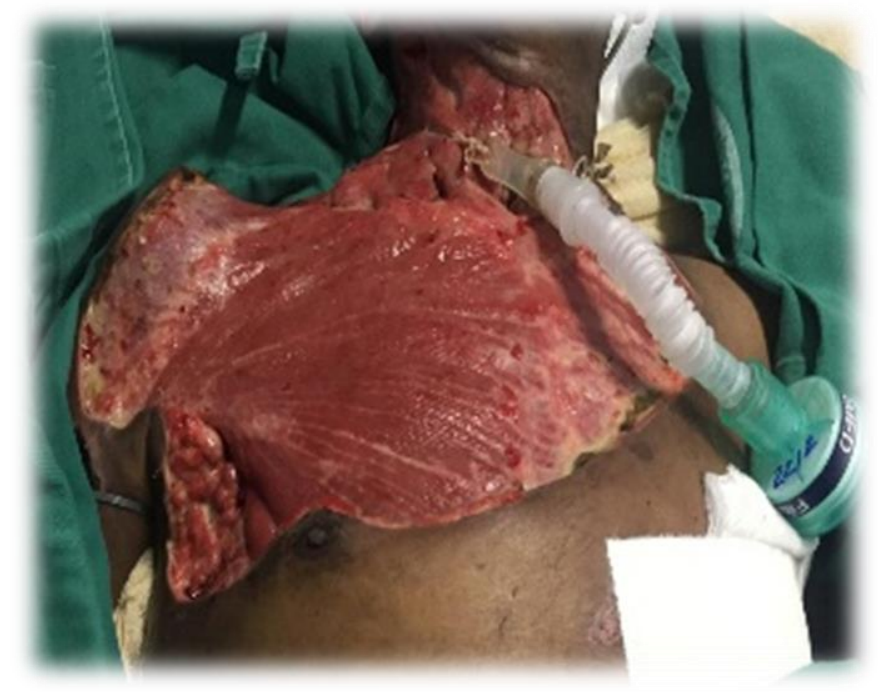

Fonte: Vasconcelos KS, et al., 2021.

Figura 6 - 90 dias após procedimento e após realização do procedimento cirúrgico de exodontia dente 46 e realização do enxerto.

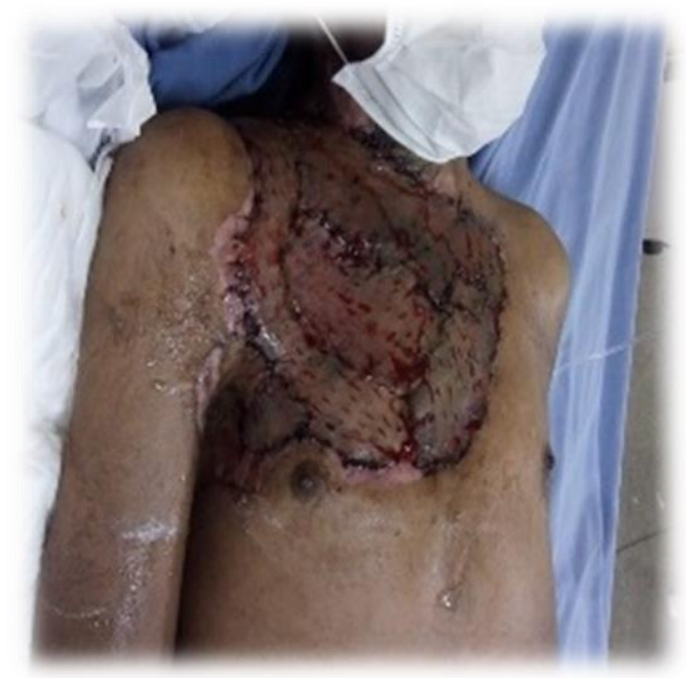

Fonte: Vasconcelos KS, et al., 2021.

\section{DISCUSSÃO}

A infecção odontogênica é considerada um problema de saúde pública, devido à morbidade causada nos pacientes e elevados custos hospitalares no seu tratamento. Originam-se nos tecidos dentais e de suporte e podem tornar-se complexas quando atingem os espaços faciais adjacentes. $O$ acometimento desta condição clinica independe de faixa etária, gênero, classe econômica ou nível de instrução (MENDONÇA JCG, et al., 2015; CAMARGOS FM, et al., 2016).

Muitos autores preconizam o tratamento das Infecções Odontogênicas com antibioticoterapia, quando ainda estão em estágio inicial. Entretanto, à medida que a condição se agrava, torna-se necessário além da antibioticoterapia, a hospitalização, drenagem cirúrgica e supervisão por equipe multiprofissional, já que estas infecções podem levar a agravamento da condição, como a obstrução das vias aéreas, infecção sistêmica disseminada, Mediastinite, Fasceite Necrosante, sepse, insuficiência múltipla de órgãos e óbito do paciente (MENDONÇA JCG, et al., 2015; JUNDT JS, et al., 2012).

A Angina de Ludwig não é uma condição frequente nos consultórios odontológicos principalmente após a era antibiótica. Mas tem sido descrita por vários autores, como uma celulite infecciosa, polimicrobiana, de 
evolução rápida e que apresenta como um dos principais sinais e sintomas: a sensação de sufocamento (falta de ar), justamente por causar edema na região de assoalho de boca e consequente elevação da língua. Febre, dificuldade de deglutição e trismo estão também presentes na avaliação clínica do paciente (EDETANLEN e SAHEEB, 2018).

A etiologia pode ser de origem odontogênica, mas a presença de comorbidades como: Diabetes Mellitus, HIV, Puerpério, gravidez, alcoolismo, desnutrição e baixa condição socioeconômicas são considerados fatores de risco para a doença. O tratamento hospitalar é o mais indicado pela necessidade de suporte emergencial ao paciente, antibioticoterapia de amplo espectro e drenagem cirúrgica (BOAMAH et al., 2019).

O diagnóstico preciso e o tratamento preventivo e precoce, evitam complicações sistêmicas ao paciente e consequente necessidade de tratamento a nível hospitalar. Quando não diagnosticada e tratada precocemente, a Angina de Ludwig pode levar a complicações, como a Mediastinite e Fasceite Necrosante (AMORIM KS, et al., 2020).

A Fasceite necrotizante consiste em ampla destruição tecidual, sendo potencialmente fatal. Na maioria das vezes é polimicrobiana. $O$ tratamento inclui diagnóstico precoce, internação para acompanhamento do paciente e administração de antibioticoterapia de largo espectro, além de intervenção cirúrgica uma vez que a remoção da causa, pode levar a um controle da infecção. Os pacientes que sobrevivem a este tipo de condição clínica, tem extensiva perda de tecido muscular e pele, necessitando na maioria dos casos, a realização de enxerto de pele para a reabilitação do paciente (GORE, 2018).

A Mediastinite é uma infecção polimicrobiana do Mediastino, de origem odontogênica em 60 a $70 \%$ dos casos. Apresenta uma taxa de mortalidade entre 37 a $60 \%$ dos casos diagnosticados. Encontra-se frequentemente associada com sepse e falência múltipla de órgãos, quando não instituído tratamento a tempo. O tratamento consiste primariamente, no debridamento da área cervical e mediastino, e administração de antibioticoterapia endovenosa (BALI et al., 2015; FUKUCHI et al., 2015).

O paciente em questão apresentou complicações resultantes de seu quadro de imunodeficiência resultante do fato de ser portador de LLA tipo B comum, além da presença de resto radicular do elemento 46, que representa um foco de infecção e contribuiu juntamente com os outros fatores supracitados, para o início e disseminação do processo infeccioso.

Sobre o atendimento odontológico a pacientes com Leucemia, o cirurgião-dentista tem papel importante no diagnóstico precoce desta condição, tendo em vista que as primeiras manifestações da doença ocorrem na boca, e os pacientes geralmente o procuram acreditando serem doenças de origem local. Procedimentos odontológicos, como raspagem periodontal, exodontias e outras cirurgias, são complicados devido às hemorragias, embora seja recomendável a remoção de focos de infecção de origem dentária para se evitar bacteremias (COSTA SS, 2011).

A higiene oral pré e pós-operatória foi realizada com Digluconato de Clorexidina $0,12 \%$; um agente antimicrobiano com amplo espectro de atividade. Indicado como um antisséptico para controle de biofilme dental e gengivite. Uma de suas propriedades está no fato de ser absorvido pelos tecidos, ocasionando um efeito residual ao longo do tempo, além de ser indicado como protocolo para higiene oral em pacientes internados em ambiente hospitalar na prevenção de Pneumonia Associada à Ventilação Mecânica (AMIB, 2013).

Vale ressaltar aqui, a importância da atuação do cirurgião-dentista junto à equipe multidisciplinar no presente caso, o que favoreceu a melhora do paciente gradativamente. A presença do cirurgião-dentista em ambiente hospitalar e nas Unidades de Terapia Intensiva (UTI) é de extrema importância e tem como objetivo principal realizar ou acompanhar a higiene oral diária dos pacientes com agente antimicrobiano, com o intuito de evitar aspiração de microrganismos via orofaringe, minimizando os riscos de pneumonia. $E$ além disto, este profissional deve estar apto ao diagnóstico e tratamento de patologias orais (BLUM DF, et al., 2018; ALOTAIBI AK, et al., 2014) 
Por fim, todo o conjunto de métodos utilizados contribuíram para um ótimo pós-operatório, com remissão de sinais e sintomas presentes das Infecções, principalmente o abscesso, oriundo do elemento 46, que obteve um excelente resultado de cicatrização logo após a exodontia.

O presente caso serve também de alerta para toda a classe odontológica: pacientes imunocomprometidos devem ser assistidos e acompanhados rotineiramente na Atenção primária em saúde ou em Centro de Referência para tratamento específico, a fim de receber tratamento odontológico preventivo e se preciso tratamento curativo, evitando assim, complicações sistêmicas como a relatada.

A outra contribuição deste trabalho para a comunidade acadêmica é apresentar e reforçar a importância do conhecimento sobre manifestações clinicas de condições sistêmicas, tomada de decisão e plano de tratamento em conjunto com equipe multiprofissional. A conscientização e sensibilização de pacientes e familiares sobre a importância do acompanhamento odontológico, faz toda a diferença para que casos como estes, sejam minoria e tenham prognóstico favorável.

A presença e assistência do cirurgião-dentista fez toda a diferença na resolução do caso, já que a etiologia das complicações da Angina de Ludwig, foi a presença de resto radicular representando a fonte de infecção principal, associado a imunodepressão sistêmica apresentada pelo paciente. Cabe ao cirurgião-dentista estar apto para atuar em ambiente hospitalar e ser capaz de junto com a equipe multidisciplinar, definir 0 diagnóstico, prognóstico e conduta para casos em que a fonte primária das complicações sistêmicas seja de origem odontogênica.

\section{REFERÊNCIAS}

1. ALOTAIBI AK, et al. Does the presence of oral care guidelines affect oral care delivery by intensive care unit nurses? A survey of Saudi intensive care unit nurses. American Journal of Infection Control, 2014; 42(8): 921-2.

2. AMORIM KS, et al. Fasceíte necrotizante de origem odontogênica na região cérvico-facial: Relato de Caso. CES Odontologia, 2020; 33(1): 30-36

3. ANTONINI MF, et al. Manifestações Orais da Leucemia no Momento do Diagnóstico. Revista Brasileira de Cancerologia, 2018; 64(2): 227-235

4. ASSOCIAÇÃO DE MEDICINA INTENSIVA BRASILEIRA (AMIB). Recomendações para Higiene Bucal do Paciente Adulto em UTI. São Paulo, 2013. Disponível em: https://www.amib.org.br/fileadmin/user_upload/amib/2018/junho/15/AMIB-Odontologia_Enfermagem-

RecomendacoesHigieneBucal-18-04-14-_Versao_2_Final.pdf. Acessado em 21/06/2021.

5. BAEZ-PRAVIA OV, et al. Should we consider IgG hypogammaglobulinemia a risk factor for severe complications of Ludwig angina?: A case report and review of the literature. Medicine, 2017; 96(47): e8708.

6. BALI RK, et al. A review of complications of odontogenic infections. National journal of maxillofacial surgery, 2015; 6(2): 136-43.

7. BHAT AA, et al. Role of non-coding RNA networks in leukemia progression, metastasis and drug resistance. Molecular cancer, 2020; 19(1): 57

8. BLUM DF, et al. A atuação da Odontologia em unidades de terapia intensiva no Brasil. Revista Brasileira de Terapia Intensiva, 2018; 30(3): 327-332

9. BOAMAH MO, et al. A comparative study of the efficacy of intravenous benzylpenicillin and intravenous augmentin in the empirical management of Ludwig's angina. Annals African Medicine, 2019, 18(2): 65-69

10. CAMARGOS FM, et al. Infecções odontogênicas complexas e seu perfil epidemiológico. Revista de cirurgia traumatologia buco-maxilo-facial, 2016; 16(2): 25-30.

11. COSTA SS, et al. Conhecimento de Manifestações Orais da Leucemia e Protocolo de Atendimento Odontológico. Revista de odontologia da Universidade Cidade de São Paulo, 2011; 23(1): 70-8.

12. DIB JE, et al. Angina de Ludwig com Evolução para Mediastinite: Relato de Caso. Revista de Cirurgia Traumatologia Buco-Maxilo-Facial, 2016; 16(4): 30-35.

13. EDETANLEN BE, SAHEEB BD. Comparison of Outcomes in Conservative versus Surgical Treatments for Ludwig's Angina. Medical Principles and Practice, 2018; 27(4): 362-366.

14. FERNANDES BR, et al. Mediastinite Pós Infecção Odontogênica: Relato de Caso. Ciência Atual, 2017; 10(2): 2-8

15. FREIRE FFP, et al. Fasceíte Necrotizante Facial Causada por Infecção Odontogênica. Revista de cirurgia traumatologia buco-maxilo-facial, $2014 ; 14(1): 43-48$.

16. FUKUCHI M, et al. Descending Necrotizing Mediastinitis Treated with Tooth Extractions following Mediastinal and Cervical Drainage. Case Reports in Gastroenterology, 2015; 9(3):311-6. 
17. GORE MR. Odontogenic necrotizing fasciitis: a systematic review of the literature. BMC Ear Nose Throat Disord, 2018; 15(18): 14-21.

18. JUNDT JS, GUTTA R. Characteristics and cost impact of severe odontogenic infections. Oral Surgery, Oral Medicine, Oral Pathology and Oral Radiology, 2012; 114(5): 558-566.

19. KIYOSHI-TEO H, BLEGEN M. Influence of Institutional Guidelines on Oral Hygiene Practices in Intensive Care Units. American Journal of Critical Care, 2015; 24(4): 309-18.

20. LOBÃO DS, et al. Condições da cavidade bucal e acompanhamento odontológico de crianças com leucemia linfocítica aguda. Revista Medica de Minas Gerais, 2008; 18(4 Supl 1): S25-S32

21. MENDONÇA JCG, et al. Infecção cervical grave de origem dentária: relato de caso. Archives of health investigation, $2015 ; 4(6): 42-46$.

22. MORAES ES, et al. Analysis of individuals with leukemia: cancer surveillance system limitations. Análise de indivíduos com leucemia: limitações do sistema de vigilância de câncer. Ciencia \& saude coletiva, 2017; 22(10): 3321-3332.

23. PROGRAMA NACIONAL DE CONTROLE DE QUALIDADE (PNCQ). Valores de Referência Hematológicos para Adultos e Crianças. In: Dacie and Lewis - Practical Haematology. 12th Edition, 2017. Disponivel em: https://www.pncq.org.br/uploads/2019/VNH2019.pdf. Acessado em 22/06/2021

24. SILVA EA, et al. A importância da atuação do cirurgião dentista na unidade de terapia intensiva: revisão integrativa. Research, Society and Development, 2020; 9(6): e164962011.

25. SOYLU E, et al. Mediastinitis as complication of odontogenic infection: A case report. The Nigerian Journal of Clinical Practice, 2019; 22(6): 869-87

26. SPREADBOROUGH P, et al. Preventing Postoperative Pneumonia Study Group and the West Midlands Research Collaborative. A systematic review and meta-analysis of perioperative oral decontamination in patients undergoing major elective surgery. Journal of Perioperative Medicine, 2016; 22(5): 6-13.

27. WANG J, et al. A five year retrospective study of odontogenic maxillofacial infections in a large urban public hospital. International Journal of Oral \& Maxillofacial Surgery, 2005; 34(6): 646-9. 